\section{Valparaíso: Miradas a un proceso de construcción socio-urbano e identitario}

Valparaíso: Glimpses to a socio-urban and identitary construction process

\section{Ricardo Molina-Verdejo}

\section{Resumen}

El presente trabajo ensaya -a partir de fuentes bibliográficas temáticamente seleccionadasacerca del proceso de construcción sociourbana de la ciudad puerto de Valparaíso (Chile). Para ello se distinguen momentos y actores centrales que dan cuenta de definiciones identitarias contemporáneas, que emergieron durante los siglos XIX y XX.

Palabras clave: Valparaíso, historia, territorio, identidad, migraciones, actores sociales, desarrollo socio-urbano.

Instituto de Historia y Ciencias Sociales, Universidad Austral de Chile. Correo electrónico: rmolina@uach.cl

\section{Abstract}

This article constitutes an essay -based on thematically selected bibliographic sourceson the process recognized as the socio-urban building of the port city of Valparaiso (Chile). To this end, central times and actors that give an account of contemporary identitarian definitions, which emerged during the $19^{\text {th }}$ and $20^{\text {th }}$ centuries, are identified.

Key words: Valparaiso, history, territory, identity, migrations, social actors, socio-urban development.

Entendemos las entidades urbanas, en general, como ámbitos que se definen y explican a través de complejos procesos y miradas. En esta dirección, cualquiera sea el análisis de caso a realizar con todas sus particularidades, deberá ser construido bajo el obligado prisma de la doble dimensionalidad en que está enmarcado: el tiempo y el espacio.

En la primera dimensión es evidente que la historicidad a la que la ciudad -como objeto de estudio- está sujeta, lleva necesariamente a comprenderla a partir de las distintas etapas que la han distinguido y caracterizado en su desarrollo, así como de los variados fenómenos que la han afectado. En este sentido, resulta fundamental la consideración de sus habitantes como protagonistas centrales vinculados con dichos fenómenos, es decir, el natural reparo de la esencia misma de la urbe. En la segunda dimensión, tanto el emplazamiento en que está localizada la ciudad como las características propias del entorno en términos del relieve, clima, flora y fauna, recursos acuíferos y afines, 
resultan claves para ayudar a comprender su desenvolvimiento, precisamente, en la historia.

Aproximarse de la mejor manera a la comprensión de lo que ha sido la vida de una ciudad como Valparaíso, supone el examen desde ámbitos disciplinarios distintos -aunque complementarios- que, en conjunto, converjan en una apreciación más integrada de sus diversas realidades. También es importante subrayar lo importante que resultan los enfoques y aspectos de interés que, desde cada disciplina, se intenta definir y trabajar en procura de aportar una pieza más al puzle del conocimiento que se pretende construir. En suma, se trata de abordar la ciudad temática y metodológicamente desde ángulos variados, con el fin de conocerla y hacerla inteligible tanto desde el punto de vista de quien vive allí, como de aquel que intenta entenderla.

Así, una de las líneas de estudio que se ha trabajado acerca de la ciudad puerto ha sido el intento de comprenderla en el contexto de los grandes procesos económicos globales que la han afectado. En efecto, los aportes realizados por la investigadora Jacqueline Garreaud (1984) han apuntado a relevar los roles que Valparaíso fue experimentando desde las primeras décadas de la centuria decimonónica, a raíz de la expansión del capitalismo comercial y financiero británico, provocando una relación de dependencia con altos y bajos en su desarrollo. La autora pone en evidencia los distintos niveles de impacto que este hecho provoca en la ciudad, incluyendo la activa presencia de una "colonia" inglesa residente como resultado y prueba de la estructura de dominio y el sentido funcional que adquiere Valparaíso como mercado de tránsito en beneficio de la economía del imperio británico.
De manera central, Garreaud (1984) destaca el papel de entrepot que alcanza el puerto en la primera mitad del siglo XIX, sobre la base de las crecientes actividades de reexportación de productos ingleses y europeos hacia mercados sudamericanos cercanos (Perú, Bolivia, Argentina y Paraguay), otorgando un gran movimiento mercantil, y generando una dinámica con múltiples beneficios a la cadena de distribución local y regional del Pacífico, los que incluyen los ingresos al Estado chileno por la vía de los tributos y pagos de derechos varios. Esta autora también da luces acerca de las exportaciones de productos chilenos a mercados tanto americanos como europeos, los que, incipientes en un principio, nutrían a los circuitos comerciales con cierta proyección.

Tampoco están ausentes de los análisis de la profesora Garreaud (1984) los conflictos de intereses y rivalidades que se producen a nivel del Pacífico Sur en el marco de la lógica descrita. Culmina su trabajo dejando a la vista el duro trance de mediados de la centuria, en donde, a partir de un repliegue de la economía británica, Valparaíso experimenta sobre la base refleja de la dependencia una fuerte crisis derivada de la saturación del mercado local y regional en cuanto a productos importados, acompañada por una ausencia severa de circulante. Todo ello trae efectos nefastos para el puerto y sus habitantes, otrora emporio del Pacífico. Con todo, gracias al surgimiento de los mercados de California y Australia por aquellos años, Valparaíso logra reactivarse nuevamente, pero siempre sujeto a los vaivenes de la inestable economía capitalista de la que seguirá dependiendo.

En síntesis, la línea de trabajo comentada logra dar cuenta de una etapa importantísima 
de Valparaíso como mercado de tránsito, habiendo considerado para ello el cuidadoso estudio y metódico análisis de las cifras de la economía portuaria tanto de salida como de entrada, más los montos que las entidades públicas registraban por diversos conceptos de servicios acordes a las actividades mercantiles. Debe destacarse el esfuerzo de la autora, como ya está dicho, por abordar también los efectos sociales y urbanos que experimentó la ciudad habida presencia de una importante colectividad inglesa residente, aunque en cierto sentido -debe decirse- también estuvo "en tránsito" dejando, no obstante, una indeleble marca en la historia porteña.

Otra interesante línea de aportes en esta materia está representada por los trabajos de los investigadores René Salinas y Baldomero Estrada, quienes, en términos generales, comparten el común interés de abordar las comunidades extranjeras residentes en Valparaíso durante los siglos XIX y XX, con el fin de ver el papel que éstas jugaron en el desarrollo del puerto tanto en lo social como en lo propiamente urbano. Conscientes del aporte de los británicos al proceso, René Salinas y Raúl Fuentes (1987) realizaron un revelador y minucioso trabajo sobre la nupcialidad al interior de la comunidad británica porteña para el período 1850-1900. Seguidamente, este trabajo fue incluido, profundizado y consolidado por Salinas (1987) en el marco de un proyecto de investigación con una vista global del siglo XIX.

Bajo los criterios metodológicos de la demografía histórica en general y del estudio de fuentes documentales ad hoc (método de reconstitución de familias), el trabajo logra reportar con detalle el comportamiento de los integrantes de la colectividad inglesa en cuanto a sus uniones matrimoniales. De este modo, queda a la vista una lógica de funcionamiento como "mercado de nupcialidad", en términos de oferta y demanda en la formación de parejas.

En un escenario en donde Valparaíso se había convertido en el principal baluarte comercial inglés en el Pacífico Sur, la existencia de una relevante agrupación de inmigrantes residentes de este origen le había otorgado a la ciudad un singular sello. Este rasgo se había traducido en un buen número de británicos que se radicaron especialmente en el Cerro Alegre. Hombres de negocios, empleados, profesionales u obreros especializados, agentes comerciales y navieros, financistas o funcionarios bancarios y similares, se habían convertido en los nuevos habitantes del puerto desde el lapso post-independista en adelante.

El estudio realizado permite advertir el fuerte sentido endogámico de la etnia al unirse sólo entre miembros de la colonia, salvo escasas ocasiones en que lo hicieron con mujeres chilenas pertenecientes a la elite local. El acto de contraer nupcias involucraba en muchos casos la unión de intereses económicos convergentes, sin perjuicio de buscar la unión entre iguales ya por razones étnicas, religiosas o culturales. En cualquier caso, el estudio deja absolutamente en claro que los actos matrimoniales tenían como resultado la mantención y la consolidación del peso relativo de la colectividad británica en la sociedad porteña decimonónica. Al parecer, la conciencia de los ingleses acerca del poder que tenían y representaban quedaba evidenciada a partir de los propios vínculos matrimoniales.

El historiador Baldomero Estrada ha desarrollado, por su parte, una fructífera y 
persistente línea de trabajo, apuntando sus esfuerzos de investigación a los estudios históricos sobre Valparaíso como entidad urbana (Estrada 2008), por un lado, y de manera paralela, a los grupos de migrantes que se fueron avecindando en el puerto tanto en el siglo XIX como en el siglo XX. En lo que a inmigraciones se refiere, ha realizado concretos aportes acerca de la colectividad italiana (Estrada 1997), española (Navarro y Estrada 2005; Estrada 2002), británica (Estrada 2006), alemana (Estrada 2011), y muy recientemente sobre la comunidad árabe(Estrada 2014).

Para el particular caso de la colectividad inglesa, es posible advertir un acabado análisis de este grupo sobre la base de tres aspectos claves: los antecedentes de la comunidad remitidos a partir del siglo XIX, la nutrida actividad comercial llevada adelante, y el significativo progreso industrial logrado en las primeras décadas del siglo XX. Para ello, el autor llevó a cabo una minuciosa revisión de fuentes archivísticas, como es el caso del Registro del Comercio de Valparaíso, y bibliográficas, tanto nacionales como internacionales, examinando, además, fuentes censales, estadísticas, periodísticas, gremiales y de viajeros, entre otras.

Baldomero Estrada, finalmente, logra dar cuenta de la prosperidad alcanzada por los británicos y su relevante contribución al desarrollo de Valparaíso y Viña del Mar, dejando en evidencia la existencia de numerosas empresas comerciales e industriales que se formaron en esos años, varias de las cuales lograron proyectarse hasta tiempos muy recientes. Destaca de manera importante, por ejemplo, el desarrollo de la industria metal mecánica, dada su imbricación con organismos del Estado como ferrocarriles, el Ejército y la
Armada, empresas navieras y otras, lo cual posibilitó un positivo impacto en el empleo de mano de obra local y extranjera. También es interesante la percepción que el estudio logra en términos de la acumulación de capital comercial y financiero en varias empresas con una presencia prácticamente exclusiva de socios británicos y, por cierto, vinculados con reconocidas familias de dicho origen que se establecieron de manera definitiva tanto en Valparaíso como en Viña del Mar.

Para el caso de la colectividad italiana, Estrada (1997) realiza su abordaje trabajando de forma inicial con el concepto de redes, a partir del cual intenta explicar el fenómeno migratorio en cuanto proceso social caracterizado por una dinámica propia. Utilizando la herramienta metodológica referida, el investigador estudia algunas variables claves como la estructura laboral que logra darse entre los italianos incorporados al mercado del trabajo local, los registros correspondientes a los establecimientos comerciales instalados (patentes otorgadas por el Fisco), especialmente almacenes medianos y pequeños que expedían alimentos y bebidas de uso cotidiano y, de modo más selectivo, empresas dedicadas a la importación de productos europeos.

En este contexto, y a la luz de los análisis realizados y los resultados obtenidos, es posible advertir la presencia de una eficaz red de intercomunicación que benefició a los italianos recién llegados al puerto. Una vez arribados, ellos se conectaban directamente con los propios paisanos residentes, quienes les facilitaban su incorporación al campo laboral controlado por los pequeños y medianos empresarios italianos del comercio local. Muchos de los inmigrantes que llegaron a 
Valparaíso en esos años eran amigos, parientes o conocidos de quienes los esperaban en Chile, por lo que el sentido de red étnico-social puede comprenderse aún mejor en este caso. Como lo han hecho saber varios autores, si bien los itálicos no disponían de una estructura de apoyo de base económica global como el caso de los ingleses, sí lograron construir un sistema solidario de apoyo para sus connacionales, favoreciendo su agregación a la sociedad local $y$ nacional de fines del siglo XIX e inicios del siglo XX.

No queda fuera del análisis sobre la colectividad italiana porteña el aporte realizado en el campo de la creación de instituciones que respondían a la importante presencia y a las necesidades de la colonia en materias educacionales, cooperativas, culturales, periodísticas, bancarias, bomberiles y deportivas, todo lo cual se tradujo en el plano urbanístico en la compra o el arriendo de propiedades y en la construcción de inmuebles. En esta dirección, Estrada (1997) destaca el acentuado interés que mostraron algunos italianos por aportar mejorías a las condiciones urbanísticas de Valparaíso, lo que evidenciaría su interés por una radicación más definitiva y no tan sólo de tránsito. Igualmente, resulta relevante la preocupación mostrada por varios italianos residentes respecto de las organizaciones sindicales, gremiales y cooperativas, lo que devino en un arraigo más eficiente en la ciudad puerto, a la vez que en un reconocimiento de la población chilena local a su aporte.

Tampoco queda excluida del estudio la nutrida actividad económica llevada adelante por algunas sociedades de la base italiana residente, en términos de la acumulación de capitales e inversiones en negocios de mayor rentabilidad, mucho más allá de los connacionales que vivían del comercio mediano o minorista a través de despachos y pulperías. En efecto, el trabajo nos muestra una importante cantidad de fortunas acopiadas y asociadas a reconocidas familias italianas, las cuales fueron invertidas o reinvertidas en negocios de nivel mayor como, por ejemplo, la minería del norte de Chile. Así, puede advertirse una directa relación entre la estratificación social lograda en el tiempo, con el nivel de empleo o actividad que se desempeñaba.

No hay que dejar de lado que tanto la colonia italiana como la española eran las más numerosas de Valparaíso en los primeros años del siglo $X X$, siendo ambas de origen latino tanto en lo cultural, como en lo étnico y en lo lingüístico, además de acreedoras de una profunda tradición católica. Dichos factores, lógicamente, debieron facilitar sus respectivas aceptaciones y adaptaciones al medio nacional. En síntesis, examinado el estudio de Baldomero Estrada hasta aquí comentado, junto con el conocimiento tanto formal como empírico que poseemos respecto de la presente temática, pocas dudas caben acerca del importante aporte realizado por los italianos a la vida social y urbana de Valparaíso, Viña del Mar y otras localidades del país.

La condición de Valparaíso de puerto marítimo principal durante los años estudiados implica, necesariamente, considerarlo como el espacio obligado para la entrada y la salida de carga y de pasajeros a nivel nacional. Al decir de Jacqueline Garreaud (1984), como ya hemos revisado, la ciudad era un entrepot en una lógica de comercio de tránsito, entendida en el contexto mundial construido por el sistema capitalista comercial británico. A partir de 
estas condiciones, Valparaíso experimentó un crecimiento económico muy relevante durante el siglo XIX, y fue destino de capitales, mercaderías y migrantes desde el exterior, habida cuenta de las crisis que se irían produciendo en la época.

Como producto de los mismos fenómenos referidos, el puerto -con sus respectivos cambios- provocó un efecto hacia el interior de Chile: se convirtió en un polo de atracción enorme desde varios puntos de vista, pero especialmente desde una perspectiva laboral. En efecto, la fuerza de trabajo nacional fue captada por Valparaíso, lo que generó una migración interna hacia la ciudad con la consabida presión sobre las fuentes de trabajo y los espacios residenciales. En este escenario, el Estado, a través de sus organismos públicos, aumentó su presencia fiscalizadora, y los municipios debieron adaptarse a las nuevas exigencias impuestas por la cambiante realidad. Ésta es la situación de fines del siglo XIX y comienzos de los primeros años del siglo $X X$. Aunque ya el escenario mundial es distinto al de 50 años atrás, Valparaíso continuó sensible a los cambios externos dado el carácter dependiente de la economía chilena, con evidentes efectos internos.

Para acercarse al estudio de la realidad social del puerto en las décadas iniciales del siglo XX -previas a la crisis del año 29-, el historiador Eduardo Cavieres (1966) realizó un trabajo cuyo objeto de estudio fueron los llamados "grupos intermedios" de la sociedad. El autor reconoce con esta categoría a aquellos trabajadores que se localizaban entre el límite de la clase obrera asalariada y los estratos más bajos de la clase media, de manera tal que cualquier variación económica podía hacerlos cambiar de nivel social. Con el propósito de definir a este grupo, el procedimiento metodológico consideró el análisis cualitativo de su sistema valórico, sus aspiraciones y las conductas asumidas vistas a través del estudio de la Sociedad de Socorros Mutuos "Asociación de Artesanos de Valparaíso", entidad que reunió a personas de diversas ocupaciones y niveles salariales. En este acercamiento a la vida de la ciudad desde la historia social, Cavieres (1966) utilizó como fuentes los Libros de Actas de la Asociación de Artesanos de Valparaíso, los registros periodísticos aparecidos en El Mercurio del puerto, junto con discursos oficiales y otros documentos vinculados a sindicatos y gremios.

Como fruto de la investigación, se advierte en el Valparaíso de aquel entonces un extraordinario movimiento a nivel de su base social, contexto en el cual, a partir de los grandes cambios generados por la economía industrial capitalista en la escala local, la clase trabajadora y obrera en general se organizó y movilizó no sólo por la protección de sus derechos, sino en procura de condiciones de mayor bienestar para ellos y sus familias. En esta lógica se entiende el mutualismo como expresión legítima de la búsqueda de esos objetivos solidarios, como así también las asociaciones gremiales y sindicales que surgieron en defensa de los intereses de sus socios frente a los síntomas gatillados por la inestabilidad económica y laboral, y por la inquietud social de la época.

Cavieres (1966) deja en evidencia las fuerzas que operaron en la sociedad porteña, las cuales obligaron a los distintos grupos de empleados, trabajadores artesanales y obreros a definirse en términos sociales. Mientras los dos primeros tendieron a identificarse con los valores cívicos impuestos por el Estado y el gobierno de turno 
en miras del ascenso social, una parte de los artesanos y los obreros se identificó con una actitud de resistencia al sistema. En ese campo agonal de energías que tensionaban a la sociedad, los grupos intermedios fueron, precisamente, el epicentro del problema, ante la disyuntiva de ser considerados al interior de un nivel social u otro.

Como se aprecia, las ciudades experimentan complejos procesos en su interior. Valparaíso no fue ni ha sido la excepción en consideración de la simultaneidad de realidades e intereses que han coexistido en el desarrollo de su historia. Más acentuadamente, desde una perspectiva disciplinaria distinta como lo es la geografía histórica, Gonzalo Cáceres y Francisco Sabatini (2007) ofrecen un sugerente trabajo a partir de la hipótesis general de la suburbanización como fenómeno explicativo del desarrollo histórico de la ciudad. Abordando el fenómeno de la suburbanización residencial desde sus primeros antecedentes hasta la actualidad, con un sentido deductivo y a partir de las experiencias fenomenológicas urbanas de otros países y ciudades, los autores analizan el concepto como efecto de los grandes procesos económicos del mundo contemporáneo.

Posteriormente, Cáceres y Sabatini (2007) comprometen sus esfuerzos en la realidad latinoamericana tomando como caso al "Gran Valparaíso" (el puerto, Viña del Mar y localidades cercanas al interior como Limache y Quillota), a partir de tres hipótesis específicas, a saber: 1) entender Viña del Mar como creación porteña, lo que sentaría las bases germinales del "Gran Valparaíso" en el siglo XIX; 2) entender que el nacimiento y la formación de Viña del Mar como ente urbano se identificaría con un suburbio y un patrón de origen angloamericano, a partir de su desarrollo en torno al ferrocarril con algunos elementos latino-mediterráneos, y; 3) la sugerencia de que Viña del Mar sería el equivalente al "Barrio Alto" de Valparaíso, en términos más o menos similares a la realidad socioeconómica santiaguina.

En un examen posterior, Cáceres y Sabatini (2007) realizan un análisis descriptivo de lo que fue el desarrollo urbano de Valparaíso y sus problemas asociados. La presencia británica vista como un esfuerzo particular en una parte de la ciudad-, junto con los serios problemas de relieve, aguas, anegamientos, incendios, terremotos, entre otros, dejaron a la vista graves obstáculos frente a los esfuerzos que hicieron organismos privados y públicos para definir la ciudad como entidad urbana. Asimismo, estos autores relevan el sello que las colectividades extranjeras residentes fueron otorgándole a sus lugares residenciales, como fueron los casos de ingleses y alemanes en los cerros Alegre y Concepción. Tras estos sellos se advierten, en suma, definiciones culturales y religiosas expresadas en la materialidad del hábitat.

El impacto que el ferrocarril entre Valparaíso y Santiago tuvo en la articulación del "Gran Valparaíso", el que comienza a definirse en la segunda mitad del siglo XIX, es también materia de análisis para los autores. La vía férrea, en efecto, en su tramo entre el puerto y Viña del Mar, permitió una integración de esta última a Valparaíso no sólo en términos físicos, sino que en el imaginario de los porteños, quienes -muchos de ellosterminaron trasladándose poco a poco hacia este incipiente "Barrio Alto". Sin perjuicio de lo anterior, una seria proyección hacia el Valle del Aconcagua gracias al ferrocarril, a propósito de las actividades agrícolas allí desarrolladas, es 
también valorada por los autores. En virtud de estos antecedentes, Cáceres y Sabatini (2007) concluyen que, paulatinamente, se encamina el proceso hacia la formación de una entidad conurbada.

Al parecer, estos autores intentan demostrar que Viña del Mar se constituye en una parte de Valparaíso en un sentido complementario, otorgándole salida a algunos problemas persistentes del puerto como la ausencia de espacio plano, por ejemplo. Esto propiciaría una migración desde el puerto tanto de extranjeros como de nacionales residentes, algunos buscando una segunda vivienda alejada de las inquietudes provocadas por la cercanía de sectores deprimidos del puerto, y otros para invertir en algún negocio o bien raíz sin abandonar Valparaíso, aprovechando para ello las bondades del tren.

El caso es que Viña del Mar experimentó importantes cambios aparejados a un sustantivo aumento de su población hacia fines del siglo XIX e inicios del siglo XX. Allí, una vez superados los problemas de ventas de tierras de las haciendas originales, se instalaron connotados personajes de la élite porteña, tanto chilenos como inmigrantes. Igualmente, surgieron importantes proyectos de inversión como la azucarera, industrias metal mecánicas y otras, como también sectores residenciales populares asociados a la mano de obra trabajadora funcional a estas nuevas instalaciones, cuyos contingentes se establecerían en conventillos dentro de lo que hoy es la céntrica avenida viñamarina Valparaíso'.

Denominación de la calle central que mira a Valparaíso en su trazado, como un símbolo de la incorporación de Viña del Mar al puerto.
En esta dirección, se advierte una clara segregación social entre un sector y otro del nuevo espacio incorporado, aunque los autores -a partir de un análisis cualitativo y cuantitativo acerca de los profesionales residentes-, concluyen que Viña del Mar, entre 1894 a 1905, estaba constituida por una población fundamentalmente de clase media, en donde la clase media-alta y alta configuraban una mayoría, en tanto que la clase media-baja y baja correspondían a una cantidad menor. Con estos antecedentes, Cáceres y Sabatini (2007) realizan un esfuerzo metodológico discutible con el fin de confirmar la hipótesis a la que finalmente adhieren, esto es, calificar a Viña del Mar como una suerte de "Barrio Alto" de Valparaíso.

También en la línea de los análisis geográficos, el trabajo de Luis Alvarez (2001) plantea aspectos que vale la pena considerar y valorar. Este autor concilia la investigación disciplinaria de base metodológica propia, con el desarrollo histórico de la ciudad vinculado a corrientes imperantes provenientes de Europa, las cuales tienen su efecto en la mentalidad y en las acciones de las personas y las autoridades. En este sentido, debe decirse que el carácter de puerto marítimo hace de Valparaíso una interesante puerta de entrada no sólo de mercaderías y pasajeros, sino que con estos últimos también de ideas e ideologías.

Pocas dudas hay respecto de las difíciles condiciones de salubridad que existieron desde siempre en el Valparaíso decimonónico, especialmente en lo que se refiere a la evacuación de las aguas servidas, y a la adecuada utilización de las vertientes de las quebradas para el uso potable del agua por parte de la población. Asimismo, la localización de ciertas actividades que atentaban contra 
la sanidad pública en pleno plan de la ciudad (mataderos), más el acopio de basurales en la orilla de la playa, ponían en peligro a la población porteña en su conjunto. La ocurrencia de más de una peste o epidemia reforzaba la idea del riesgo existente a partir de las mal llamadas miasmas.

A partir de los discursos higienistas que se impusieron en ciertos sectores de la sociedad, compartidos por las autoridades arribadas desde Europa y basados en los socialismos utópicos igualitarios, se propuso la distribución justa y equitativa a toda la población de recursos como el agua. De este modo, en la segunda mitad del siglo XIX, se implementaron diversas obras públicas con el propósito de lograr abovedar los cauces provenientes de las quebradas porteñas. Con la colaboración de agentes privados y públicos, la propia Municipalidad de Valparaíso tomó cartas en el asunto, lo que no estuvo exento de problemas, particularmente en lo referido al quehacer que tuvo en esta materia el ciudadano y empresario estadounidense Guillermo Wheelwright ${ }^{2}$, pues, en el intento por instalar una red de agua potable en el sector plan, terminó produciéndose un conflicto con el municipio local.

Sobre la base de estas obras de canalización fue posible definir nuevos espacios públicos en favor de la población, sobre la base de una red de cauces que facilitó la escorrentía de las aguas y el aprovechamiento de ellas, lo que fue acompañado por la habilitación de espacios que posteriormente fueron reconocidos como "parques" (el caso del Parque Italia es uno), equivalentes a las plazas. Resulta evidente que esto logró dar cierta direccionalidad y ordenamiento a la parte plana de la ciudad, lo que en los años siguientes fue reforzándose con obras mayores que permitieron ganar más espacios al mar, sumándolos al plan de Valparaíso.

A modo de reflexión final, la sensación que nos queda se vincula directamente con haber intentado construir conocimiento acerca de un singular proceso: el desarrollo de la ciudad de Valparaíso en periodos claves de su formación y la participación de algunos de los actores protagónicos en este cometido. Ello, sin dudarlo, nos permite aprehender mejor a la ciudad y a su gente, a la vez que nos acerca al sentido identitario que ésta posee y que la hace única y distinta a cualquier otra de características similares. Ciertamente, como ciudad puerto, Valparaíso contiene en su alma social y urbana algo de todo el mundo y, al mismo tiempo, el mundo tiene algo representado en él. En efecto, sin perjuicio de enfrentarnos a una sociedad nacional de base históricamente mestiza, el acto de comprender a Valparaíso debe ir asociado no sólo a sus rasgos físicourbanísticos tan particulares de acuerdo a los atributos del espacio geográfico en que fue asentada la ciudad desde su fundación, sino que, de manera central, a lo que fue su construcción multicultural y multiétnica en los últimos dos siglos de cara al Pacífico Sur.

"Demanda de nulidad del Cabildo de Valparaíso que prohíbe a la Compañía de Consumidores de Agua tender sus cañerías en las calles de la ciudad". En Dictámenes del Consejo de Estado. Policía i uso de las calles i vías públicas. Dictámenes del Fiscal de la Corte Suprema de Chile, Ambrosio Montt. Materias judiciales i Administrativas. Santiago de Chile, 1890. 


\section{Bibliografía}

Alvarez, Luis. 2001. "Origen de los espacios públicos en Valparaíso: El discurso higienista y las condiciones ambientales en el siglo XIX". Revista de Urbanismo 4. En línea, disponible en: http://www.revistaurbanismo.uchile. cl/index.php/RU/article/viewFile/11804/12167

Cáceres, G. y Sabatini, F. 2007. "Suburbanización y segregación urbana en Chile decimonónico. Hipótesis sobre la formación histórica del Gran Valparaíso". Historias urbanas. Homenaje a Armando de Ramón. Valenzuela, J. (Ed.). Santiago: Ediciones Pontificia Universidad Católica de Chile. 93-121.

Cavieres, E. 1966. "Grupos intermedios e integración social: La sociedad de artesanos de Valparaíso a comienzos del siglo XX". Cuadernos de Historia 6: 33-47.

Estrada, B. 2014. "Desarrollo empresarial inmigrante. La colectividad árabe en Valparaíso (1900-1940)". Interciencia 39 (12): 850-856.

2011. "Integración socio-económica de la colectividad alemana en Valparaíso (1850-1930)". Historia 3961 (2): 199-235.

2008. "Identidades históricas de Valparaíso patrimonial: Función portuaria durante la primera mitad del siglo XX". Valparaíso. Patrimonio arquitectónico, social y geográfico. Estrada, B. (Comp.). Viña del Mar: Ediciones
Altazor. 25-52.

2006. "La colectividad británica en Valparaíso durante la primera mitad del siglo XX". Historia 39 (1): 65-91. 2002. "La historia infausta de la inmigración española en Chile a través de los conflictos comerciales y políticos". Historia 35: 63-89.

1997. "Redes socioeconómicas y mercados urbanos: La colectividad italiana de Valparaíso en el cambio de siglo". Estudios Migratorios Latinoamericanos 12 (35): 153-185.

Garraud, J. 1984. "La formación de un mercado de tránsito. Valparaíso: 1817-1848". Nueva Historia. Revista de Historia de Chile 3 (11): 157-193.

Navarro, C. y Estrada, B. 2005. "Migración y redes de poder en América: El caso de los industriales españoles en Valparaíso (Chile) 1860-1930". Revista Complutense de Historia de América 31: 115-146.

Salinas, R. y Fuentes, R. 1987. Estudio de nupcialidad en la comunidad británica de Valparaíso: 1850-1900. Valparaíso: Universidad Católica de Valparaíso.

"Nupcialidad, familia y funcionamiento del mercado matrimonial en Valparaíso durante el siglo XIX". Valparaíso 1536-1986. V.V. A.A. Viña del Mar: Editorial Altazor. 77-84. 